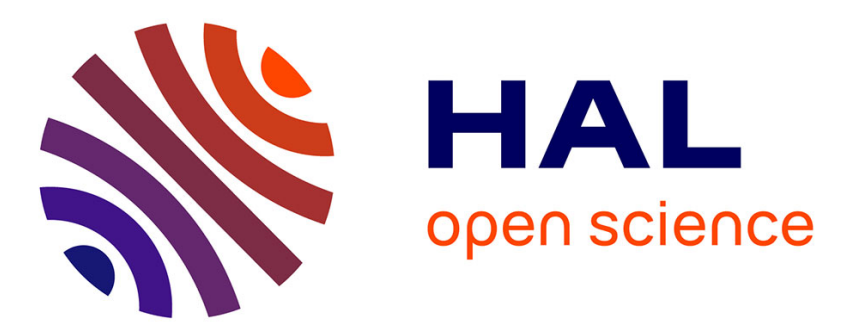

\title{
Coordinate Free Approach for the Calculation of Geometrical Requirement Variations
}

Guillaume Mandil, Philippe Serré, Alain Desrochers, André Clément, Alain Rivière

\section{To cite this version:}

Guillaume Mandil, Philippe Serré, Alain Desrochers, André Clément, Alain Rivière. Coordinate Free Approach for the Calculation of Geometrical Requirement Variations. 20th CIRP Design, Apr 2010, Nantes, France. hal-00482274

\section{HAL Id: hal-00482274 https://hal.science/hal-00482274}

Submitted on 10 May 2010

HAL is a multi-disciplinary open access archive for the deposit and dissemination of scientific research documents, whether they are published or not. The documents may come from teaching and research institutions in France or abroad, or from public or private research centers.
L'archive ouverte pluridisciplinaire HAL, est destinée au dépôt et à la diffusion de documents scientifiques de niveau recherche, publiés ou non, émanant des établissements d'enseignement et de recherche français ou étrangers, des laboratoires publics ou privés. 


\title{
Coordinate Free Approach for the Calculation of Geometrical Requirement Variations.
}

\author{
Guillaume MANDIL ${ }^{1,2}$, Philippe SERRÉ ${ }^{2}$, Alain DESROCHERS ${ }^{1}$, André CLEMENT ${ }^{3}$, Alain RIVIĖRE ${ }^{2}$ \\ ${ }^{1}$ Université de Sherbrooke, Département de génie mécanique, \\ 2500 boulevard de l'université, Sherbrooke Québec, Canada J1K 2R1 \\ ${ }^{2}$ LISMMA - Supméca Paris, 3 rue Fernand Hainaut, F-93407 Saint-Ouen Cedex, France \\ ${ }^{3}$ Dassault Systèmes, 10 Rue Marcel Dassault, F-78140 Vélizy-Villacoublay, France
}

Guillaume.Mandil@Usherbrooke.ca

\begin{abstract}
This paper proposes to investigate the use of a coordinate free approach for the mapping of geometrical requirement along a product life cycle. The geometry of the studied assembly is represented using a Gram matrix that is issued from a parametric model constituted of points and vectors. This parametric model is instanced for all relevant phase of the product life cycle. The calculation of instanced parameters is based on part deformation due to changing operating conditions. This calculation is carried out thanks to existing theoretical techniques. The application presented in this paper is constituted of a simple 3D case composed of 3 articulated bars disposed as a tetrahedron and subjected to some thermal expansion.
\end{abstract}

Keywords:

Geometrical requirement, PLM, Life Cycle, Part deformation, TTRS, Metric tensor, Gram matrices, Non-Cartesian geometry.

\section{INTRODUCTION}

In most cases, a mechanical product is subjected to thermo-mechanical loads which vary along its life cycle. These variations are inducing elastic deformations, which in turn can influence the value of the functional or geometrical requirements. Generally the useful value of a requirement is the one taken under operating conditions. However, the great majority of products are designed and represented in Computer Aided Design (CAD) systems at the assembly stage of their life cycle. Moreover, the contractual drawings are generally specified at ambient temperature. This means that in fact, the values of geometrical or functional requirements are not necessarily calculated, specified and checked under operating conditions. As a matter of fact, considering the designer point of view, several problems appear: "Does the chosen dimension allows to meet the requirements under operating conditions?" or "Which dimensions must be specified on the drawing to ensure a given value of the functional requirement in operation?"

A typical application that illustrates best the above idea would be that of a jet engine for which the functional requirement varies during its own lifecycle. Indeed, the clearance between the rotor blades and engine housing (or stator) of the turbine will be quite different at assembly and in operation due to the high temperature and rotation velocity to which the rotor is subjected in service. In this case, the above-mentioned designer problem should be: "Does the actual dimensions of the blades and the stator housing of the turbine ensure that interference under operating conditions will not appear?" and "Which dimension have to be specified at assembly to avoid any interference between the blade and the stator in operation?"

In order to provide the designer with proper tools for this kind of issue, this paper investigates the suitability of a coordinate free approach based on Gram matrix for the calculation of a functional or geometrical requirement along the product life cycle. To effectively take into account the dimensions evolution along the product life cycle, the proposed approach integrates part deformation (as calculated from the stress analysis) as a variation of the parameters of the model.

This paper will introduce previous scientific contributions done in this field of research. Afterwards the coordinate free parametric model used in this research will be exposed and then an application case will be detailed.

\section{LITERATURE REVIEW}

This section presents first some prior work done for the tolerancing of parts subjected to deformations. Afterwards geometrical models used to build the presented coordinate free approach are introduced. Finally, this section will conclude with the introduction of two configuration management concepts inspired from the $\mathrm{PLM}^{\top}$ community that might be useful for complex cases.

\subsection{Tolerancing flexible parts}

Researches that have caught the authors' attention will now be presented. Firstly, Samper [1][2] presents an approach which considers the influence of both part deformation and fit of joint into the analysis or synthesis of tolerance zones. Secondly, Cid [3] developed a model which permits the evaluation of clearances under loads using a clearance torsor introduced in [4]. This study investigates the case of the clearance between a vehicle door and its frame. The representation of parts considers $3 D$ surfaces instead of 3D volumes. Finally, Pierre [5][6] has investigated part of the jet engine particular issue mentioned in the introduction.

\footnotetext{
${ }^{1}$ Product Life-cycle Management
} 


\subsection{Geometrical representations}

Desrochers [7] proposed the TTRS ${ }^{2}$ model which is based on a binary and recursive association of two functional surfaces (or group of surfaces). Globally, the goal of this association is to link each functional surface to another. The result of the association process on all the functional surfaces of a part or a mechanism is generally represented as a hierarchy tree. Additionally, this approach uses the concept of $\mathrm{MGRE}^{3}$ to obtain a mathematical representation of a given TTRS. The hierarchy tree is constructed by going through independent cycles in the kinematic graph. This theory is detailed in [7][8][9].

Serré [10] proposes a theory to specify univocally a geometrical problem. This is based on an information model that ensure the coherence of the specifications made by the designer and on the specification of univocal constraints between surfaces. Afterwards, in order to obtain the solution to the problem, these specifications and constraints are implemented in a coordinate free geometrical model (based on the Gram matrix [11]) to generate the algebraic relations to be solved. According to Serré this model is appropriate to describe both geometrical and technological problems. In this work Serré uses the TTRS/MGRE model to describe the relative spatial positions of specifications in $3 \mathrm{D}$ vector space. This research proposes to use this theory for the specification and resolution of geometrical requirements.

\subsection{Configuration management}

In the field of Product Life-cycle Management (PLM), some researchers have introduced interesting concepts like Zina [12] who defined the concept of "context" which could be used to define loads and environment in the proposed approach. Alternatively Eynard [13] presents an object-oriented approach to help the designing team with the transmission of both design and calculation data such as geometry, use cases, loads, etc.

\section{MODELS USED}

\subsection{Context}

As previously explained in [14] and [15] functional or geometrical requirements will be taken into account considering the evolution of their mean value along the product life cycle as presented in figure 1.

\begin{tabular}{|c|c|}
\hline Life-cycle stage & 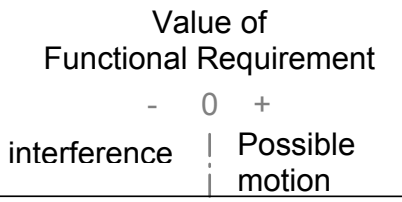 \\
\hline Stage "Si" & $\underbrace{}_{\Delta j 1}$ \\
\hline
\end{tabular}

Figure 1: Functional requirement values evolution along the product life cycle

\footnotetext{
${ }^{2}$ Technologically and Topologically Related Surface

${ }^{3}$ Minimum Geometrical Reference Element
}

Indeed if the width of the tolerance zone (noted $\Delta j 1$ in figure 1 ) is small compared to the dimension itself, then at a first order of approximation, the variations of the tolerance zone width will be negligible with regard to the variations of the dimension itself. This is why the width of the tolerance zone remains unchanged in figure 1 whereas the load variation induce a shift of the mean value (noted $\Delta \overline{j 1}$ in figure 1 ) of the geometrical (or functional) requirement.

However, it remains necessary to include tolerance zones in the model as they provide the designer with intervals for the possible value of a requirement (due to machining uncertainties). If necessary, the width of the interval can be calculated by using existing techniques of tolerance analysis or synthesis [16][17][18][19].

The geometric dimensioning problem addressed in this paper is the coupling of dimensions (or geometric parameters) with one or several physical effects. More specifically, as mentioned in the introduction the goal of this research is to evaluate the variation of functional or geometrical requirements due to the changing environment. This paper proposes to use a topological connector between the geometrical vectors space and some physicals vectors spaces.

\subsection{Coupling several physical effects through the topology of their vector space}

In our study, we suppose that the topology of the mechanism remains unchanged during the shift from one stage of the product life cycle to another. The topology and the geometrical (or functional) requirements define some relations between geometrical elements. In order to meet user's expectations, these relations have to be satisfied. If we consider two configurations of the same product at two different stages of its own life cycle, the topological and functional relations remain the same for both configurations. Only the values of the parameters involved in these relations are changing.

As a fist step, the authors propose to map geometrical requirement evolution along the relevant stages of the product life cycle. This means that, firstly the calculation inputs are topology, initial values of dimensional parameters, and loads and the result is the value of the geometrical requirement under the considered loads. This approach is a form of geometrical requirement analysis along the product life cycle.

\subsection{Proposed approach}

This paper proposes to investigate the use of a coordinate free approach for the calculations of the geometrical requirement evolution along the product lifecycle. With this approach, for a given geometry described in different coordinate systems, the resulting coordinate free description would be exactly the same whatever the initial coordinate systems may have been.

This choice has been motivated by the existence of generic constraints specification techniques (like in [10]) and solvers (cf. in [20]) to resolve these constraints. Authors decided to use a Gram matrix (presented below) as the chosen mathematical representation of the mechanism.

As this approach is vector space based, it becomes necessary to obtain vectors to represent the product model. There exists several ways to obtain these vectors. Among these possibilities it is conceivable to use the TTRS/MGRE model to obtain the relative position of the technological surfaces. From there it becomes possible to extract one or several vectors to represent the relative positions of two surfaces, parts or components. 
Moreover, during the early stages of the design of a product, there often exists a simple geometrical representation of the product such as a skeleton from which positioning vector parameters will be extracted.

\subsection{Mathematical tools}

\section{Gram matrices theory}

The following definition is found in [11] : «the Gram matrix (or Gramian matrix or Gramian) of a set of vectors $\left\{\mathbf{x}_{1}, \mathbf{x}_{2}, \ldots, \mathbf{X}_{\mathbf{k}}\right\}$ in an inner product space is the Hermitian matrix of inner products, whose entries are given by equation (1)». In this paper the names of all Gram matrices start with a "G". Gramian matrix are always square (same number of rows and columns).

$\mathrm{G}_{\mathrm{Im}}=\left\langle\mathbf{x}_{\mathbf{l}}, \mathbf{x}_{\mathrm{m}}\right\rangle$

A metric tensor is a particular case of a Gram matrix which has its rank equal to its dimension. For example, in the 3D Euclidian space, a 3 by 3 Gram matrix which has a nonzero determinant (which also means the matrix is constituted of 3 independent vectors) is called a metric tensor. In this paper the names of all metric tensor start with an "M".

\section{Application to assemblies}

In any case, this paper supposes that a vectorial representation of the assembly already exists. The vectors included in this representation will be noted un where $\mathrm{n}$ represents the current life-cycle stage and $I$ is an index used to count the vectors. For example at the assembly stage noted $n$ of the product life cycle, the set of $\mathrm{k}$ vectors is noted : Sun $=\left\{\mathbf{u a}_{\mathbf{1}}, \mathbf{u} \mathbf{a}_{\mathbf{2}}, \ldots, \mathbf{u} \mathbf{a}_{\mathbf{l}}, \ldots, \mathbf{u} \mathbf{a}_{\mathbf{k}}\right\}$.

From this set of vector, and for each configuration of the product, the assembly is represented by its associated Gram matrix which will be noted $\mathbf{G}$ n where $\mathrm{n}$ represents the current life-cycle stage.

\section{Geometrical requirement calculation}

Functional requirements are expressed as an algebraic relation between the vectors (or combination of vectors) used in the Gram matrix. Basically it's immediately possible to obtain the scalar product between two vectors. From there it's easy to deduce the angle between two vectors and the norm of a vector.

More generally, the geometrical requirement (noted grn at stage $n$ ) might be expressed thanks to a linear combination of scalar products between vectors included in the set Sun above mentioned and presented in equation (2) where the two vectors $\beta$ and $\gamma$ represent the weight of $\mathbf{G n}$ in grn..

$$
\begin{aligned}
\text { grn } & =\sum_{I, m=1}^{k}<\beta_{l} \mathbf{u n}_{\mathbf{l}}, \gamma_{\mathrm{m}} \mathbf{u n}_{\mathbf{m}}> \\
& =\sum_{I, m=1}^{k} \beta_{l} \mathrm{Gn}_{\mathrm{Im}} \gamma_{\mathrm{m}}
\end{aligned}
$$

Reworking equation (2) in a matrix form yields equation (3):

$$
\operatorname{grn}=\beta \cdot G_{n} \cdot \gamma^{\top}
$$

\section{Link between configurations}

In order to map the evolution of the geometrical requirement along the life cycle from an initial stage $i$ to a final stage $f$ it become necessary to propose a way to link (or superpose) the two relevant configurations. A vectorial association of the two configurations is required in order to define their relative orientation. The $\mathbf{G i}$ and $\mathbf{G f} \mathrm{Gram}$ matrices ( $\mathbf{G i}$ stand for the Gram matrix corresponding to the initial stage and $\mathbf{G f}$ for the Gram matrix corresponding to the final stage) are supposed to be known and the goal of the association is the calculation of a relative orientation matrix Gif such as that defined in equation (4).

$\mathrm{Gif}_{\operatorname{lm}}=\left\langle\mathbf{u i}_{\mathrm{I}}, \mathbf{u f}_{\mathrm{m}}\right\rangle$

From there it become possible to build a Gram matrix $\mathbf{G}$, expressed in equation (6), including all the vectors from the two configurations that define the set Suif as expressed in equation (5).

$$
\begin{aligned}
& \text { Suif }=\left\{u i_{1}, \ldots u i_{k}, u f_{1}, \ldots, u f_{k}\right\} \\
& =\left\{\text { uif }_{1}, \text { uif }_{2}, \ldots, \text { uif }_{\text {m }}\right\} \\
& \mathrm{G}_{\mathrm{Im}}=\left\langle\text { uif }_{\mathrm{l}}, \text { uif }_{\mathrm{m}}\right\rangle
\end{aligned}
$$

Moreover, in order to calculate a point deviation it's necessary to associate the configuration in an affine way. This association basically consist in assuming that a point in the two configurations has a known deviation (that could be null) during the shift from stage $i$ to stage $f$.

\section{Relations between two configurations}

The above section pointed out the necessity of an association between the relevant configurations of a mechanism. To carry out this association it is necessary to calculate the "relative orientation Gram matrix" described in equation (4). The direct application of equation (4) is impossible because the Gram matrix approach is coordinate free. Therefore, the relative orientation has to be calculated using another technique.

Authors propose to use matrix factorisation techniques to express any $\mathbf{G n}$ as a product specified in equation (7) where Id stands for the identity matrix.

$\mathbf{G n}=\mathbf{F n}^{\top} \cdot \mathbf{F n}=\mathbf{F} \mathbf{n}^{\top} \cdot \mathbf{I d} \cdot \mathbf{F n}$

Relation (7) is equivalent to (8) using Enstein's convention.

$$
\mathrm{Gn}_{\mathrm{Im}}=\mathrm{Fn}_{\mathrm{pl}} \cdot \mathrm{Fn}_{\mathrm{pm}}=\mathrm{Fn}_{\mathrm{pl}} \cdot \delta_{\mathrm{pq}} \cdot \mathrm{Fn}_{\mathrm{qm}}
$$

In the relation (7), Id can be viewed as the metric tensor of an ortho-normal basis noted $\mathbf{S e}\left\{\mathbf{e}_{1}, \mathbf{e}_{2}, \mathbf{e}_{3}\right\}$. From there $\mathrm{Fn}^{\top}$ can in turn be viewed as the transformation matrix defined in (9). As $\mathbf{G n}$ is a Gram matrix that is positive semidefinite, the terms of Fn are Real numbers.

$\mathrm{un}_{\mathrm{I}}=\mathrm{Fn}_{\mathrm{lq}} \cdot \mathrm{e}_{\mathrm{q}}$ with $n=i$ or $n=f$

The application of relation (9) on the terms of relation (4) Sui and Suf (as defined in section 3.4) finally gives the expression (10) that allows the calculation on Gif.

$$
\begin{aligned}
\mathrm{Gif}_{\mathrm{Im}} & =\left\langle\mathrm{Fi}_{\mathrm{I}} \bullet \mathrm{e}_{\mathrm{p}}, \mathrm{Ff}_{\mathrm{qm}} \cdot \mathrm{e}_{\mathrm{q}}>=\mathrm{Fi}_{\mathrm{Ip}} \cdot \mathrm{Ff}_{\mathrm{mq}}<\mathrm{e}_{\mathrm{p}}, \mathrm{e}_{\mathrm{q}}>\right. \\
& =\mathrm{Fi}_{\mathrm{Ip}} \cdot \mathrm{Ff}_{\mathrm{mq}} \bullet \delta_{\mathrm{pq}}=\mathrm{Fi}_{\mathrm{Ip}} \cdot \mathrm{Ff}_{\mathrm{mp}}
\end{aligned}
$$

Currently, authors have envisaged the use of two factorization techniques:

- A singular value decomposition

- A Cholesky based factorisation

The affine association consists simply in an appropriate choice of a point to connect a path from the initial to the final configuration. The application on the case in the following section will clearly explicit this.

\section{APPLICATION CASE: 3 ARTICULATED BARS IN 3D}

This section proposes an application of the techniques presented above.

\subsection{Case description}

This subsection presents the case that has been chosen for the illustration of the method. The following subsections introduce elements such as: the initial geometry, the topology, the initial gram matrix, the studied geometrical requirements, the physical effect that 
rules the part deformation and the geometrical requirement evolution.

\section{Initial geometry}

The proposed model for this study is composed of three articulated bars disposed as a tetrahedron on a wall such as presented in figure 2 .

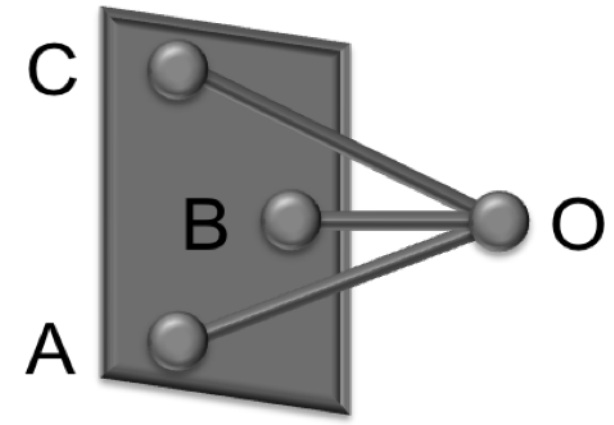

Figure 2: Initial geometry of the case study.

The four points constituting the structure $\{O, A, B, C\}$ have their initial coordinates in a global ortho-normal coordinate system (noted $\{\mathbf{e i}, \mathbf{e j}, \mathbf{e k}\}$ )presented in table 1 below. This coordinate matrix is noted $\mathbf{X O}$.

\begin{tabular}{|c|c|c|c|}
\hline Point & $\begin{array}{c}\text { X coordinate } \\
\text { (along ei) }\end{array}$ & $\begin{array}{c}\text { Y coordinate } \\
\text { (along ej) }\end{array}$ & $\begin{array}{c}\text { Z coordinate } \\
\text { (along ek) }\end{array}$ \\
\hline O & 0 & 0 & 0 \\
\hline A & 1 & 0 & 0 \\
\hline B & 0 & 1 & 0 \\
\hline C & 0 & 0 & 1 \\
\hline
\end{tabular}

Table 1: Matrix X0 of initial point coordinates in a global reference system.

\section{Case topology}

The topological diagram of the truss is defined using 4 vertex and 6 edges. Three of these edges are the bars represented in figure 2 and the three others are used to define the support wall $\{A B C\}$. The connexion between points and bars is done thanks to a connection matrix (noted $\mathrm{C} 0$ ) which rows contains the six bars and which columns contains the four $\{O, A, B, C\}$ points. This matrix is presented in table 2.

\begin{tabular}{|c|c|c|c|c|}
\hline & O & A & B & C \\
\hline oa & -1 & 1 & 0 & 0 \\
\hline ob & -1 & 0 & 1 & 0 \\
\hline $\mathbf{o c}$ & -1 & 0 & 0 & 1 \\
\hline $\mathbf{a b}$ & 0 & 1 & -1 & 0 \\
\hline $\mathbf{a c}$ & 0 & 1 & 0 & -1 \\
\hline $\mathbf{b c}$ & 0 & 0 & -1 & 1 \\
\hline
\end{tabular}

Table 2: Connectivity Matrix C0

\section{Initial coordinate free representation.}

As explained in section Erreur! Source du renvoi introuvable. the coordinate free approach use in the paper uses the Gram matrix that is vector based. This model induces intuitively the choice of the six vectors of the edges presented in table 2. The Gram matrix $\mathbf{G i}$ associated to the initial configuration is calculated using equation (11).

$\mathbf{G i}=\left(\mathrm{XO}^{\top} \cdot \mathrm{CO}^{\top}\right) \cdot\left(\mathrm{XO}^{\top} \cdot \mathrm{CO}^{\top}\right)^{\top}$

The numerical values of $\mathbf{G i}$ are presented in table 3 .

\begin{tabular}{|c|c|c|c|c|c|c|}
\hline & $\mathbf{o a}$ & $\mathbf{o b}$ & $\mathbf{o c}$ & $\mathbf{a b}$ & $\mathbf{a c}$ & $\mathbf{b c}$ \\
\hline $\mathbf{o a}$ & 1 & 0 & 0 & -1 & -1 & 0 \\
\hline $\mathbf{o b}$ & 0 & 1 & 0 & 1 & 0 & -1 \\
\hline $\mathbf{o c}$ & 0 & 0 & 1 & 0 & 1 & 1 \\
\hline $\mathbf{a b}$ & -1 & 1 & 0 & 2 & 1 & -1 \\
\hline $\mathbf{a c}$ & -1 & 0 & 1 & 1 & 2 & 1 \\
\hline $\mathbf{b c}$ & 0 & -1 & 1 & -1 & 1 & 2 \\
\hline
\end{tabular}

Table 3: initial Gram matrix Gi

Geometrical requirements definition

For this study, authors arbitrarily propose to take into account as geometrical requirements, the deviation of the vertex $\mathrm{O}$ and the scalar products <0o', oa $\rangle,\langle\mathbf{0 0}, \mathbf{o b}\rangle$ and <oo',oc>. O' correspond to $O$ in the deformed configuration in accordance with section 4.2.

Physical effect: thermal expansion

In accordance with the objective exposed in section 3.2, authors decided to study the effect of a thermal expansion of the structure. This physical effect has been chosen for the ease of its theoretical formulation as given in equation (12). In expression (12) li and If stands respectively for initial and final length of the bars (vectors). In the same way $t i$ and $t f$ are representing the initial and final temperature and $\alpha$ refers to the thermal expansion coefficient (typical values of $\alpha$ are found in the literature).

If $=\alpha \times l i \times(t f-t i)+l i$

Authors assume that the wall of figure 2 is not subjected to thermal expansion, consequently the value of $\alpha$ is set to zero for vectors $\mathbf{a b}, \mathbf{a c}$ and $\mathbf{b c}$. Moreover, the value of $\alpha$ is set at $3 \mathrm{E}-05 \mathrm{~K}^{-1}$ for $\mathbf{0 a}$, ob and oc vectors.

It's also supposed that the initial temperature is $20^{\circ} \mathrm{C}$ and the final temperature is $100^{\circ} \mathrm{C}$ for all the bars involved in the configuration.

Thanks to equation (1), the values of the initial lengths (li) of the bars (or the initial norm of the vectors) are directly deduced from the square root of the diagonal terms of $\mathbf{G i}$ (see in table 3). For example: $\|\mathbf{o a}\|=\sqrt{\langle\mathbf{o a}, \mathbf{o a}\rangle}=\sqrt{\mathrm{Gi}_{1,1}}$.

With these hypotheses and the formulation of (12) it become possible to calculate the value of the final length (If) of the bars (or the final norm of the vectors).

A summary of these assumptions and the result of the final length calculations are presented in table 4 below.

\begin{tabular}{|c|c|c|c|c|c|}
\hline Vector & $\alpha$ & $t i$ & $t f$ & li & If \\
\hline oa & $3 \mathrm{E}-05 \mathrm{~K}^{-1}$ & \multirow{6}{*}{$20^{\circ} \mathrm{C}$} & \multirow{6}{*}{$100^{\circ} \mathrm{C}$} & 1 & 1.0024 \\
\hline ob & $3 \mathrm{E}-05 \mathrm{~K}^{-1}$ & & & 1 & 1.0024 \\
\hline OC & $3 \mathrm{E}-05 \mathrm{~K}^{-1}$ & & & 1 & 1.0024 \\
\hline$a b$ & 0 & & & $\sqrt{2}$ & $\sqrt{2}$ \\
\hline ac & 0 & & & $\sqrt{2}$ & $\sqrt{2}$ \\
\hline bc & 0 & & & $\sqrt{2}$ & $\sqrt{2}$ \\
\hline
\end{tabular}

Table 4: value for thermal expansion calculation

Geometrical requirements

Authors propose to consider the position of the vertex $O$ as the geometrical requirement under study. The mapping of its evolution will be done with the measurement of the norm of the displacement vector of vertex $O$. This vector is noted oo' and its norm is calculated with: $\left\|\mathbf{O O ^ { \prime }}\right\|=\sqrt{\left\langle\mathbf{O O}^{\prime}, \mathbf{O O}^{\prime}\right\rangle}$. Moreover, in order 
to compare the results with a Cartesian method, the

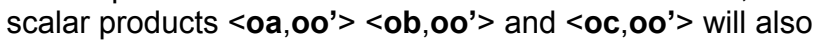
be computed.

\subsection{Calculations and results}

\section{Gram matrix of the final configuration}

First of all, the reader is advised that the points in the final configuration (represented by the Gram matrix Gf) are expressed with primes. For example, the vertex $O^{\prime}$ in the final configuration corresponds to the initial vertex $\mathrm{O}$. In accordance with section 4.1 , the displacement vector of vertex $\mathrm{O}$ is noted oo'.

Moreover, relation (1) allows the direct calculation of the diagonal terms of the $\mathbf{G f}$ matrix. These values are directly the square of the final lengths presented in the If column of table 4. For example $\mathrm{Gf}_{1,1}=<\mathbf{o}^{\prime} \mathbf{a}^{\prime}, \mathbf{o}^{\prime} \mathbf{a}^{\prime}>=\left\|\mathbf{o}^{\prime} \mathbf{a}^{\prime}\right\|^{2}$.

From there, it becomes possible to calculate all the others terms of the matrix using the Chasles relation as in (13).

$<\mathbf{u}, \mathbf{v}\rangle=(\langle\mathbf{u}+\mathbf{v}, \mathbf{u}+\mathbf{v}\rangle-\langle\mathbf{u}, \mathbf{u}\rangle-<\mathbf{v}, \mathbf{v}\rangle) / 2$

For example the value of <o'a',o'b'> is given by equation (14) below which uses only the diagonal terms of $\mathbf{G f}$.

$$
\begin{aligned}
<0^{\prime} a^{\prime}, o^{\prime} b^{\prime}> & =-<a^{\prime} o^{\prime}, o^{\prime} b^{\prime}> \\
& =-\left(<a^{\prime} b^{\prime}, a^{\prime} b^{\prime}>-<a^{\prime} 0^{\prime}, a^{\prime} o^{\prime}>-<0^{\prime} b^{\prime}, o^{\prime} b^{\prime}>\right) / 2
\end{aligned}
$$

In the end, the $\mathbf{G f}$ matrix expression is given in table 5 below.

\begin{tabular}{|c|c|c|c|c|c|c|}
\hline & o'a' & o'b' $^{\prime}$ & o'c' & a'b' $^{\prime}$ & a'c' $^{\prime}$ & b'c' \\
\hline o'a' & 1.0048 & 0.0048 & 0.0048 & -1 & -1 & 0 \\
\hline o'b' & 0.0048 & 1.0048 & 0.0048 & 1 & 0 & -1 \\
\hline o'c' $^{\prime}$ & 0.0048 & 0.0048 & 1.0048 & 0 & 1 & 1 \\
\hline a'b' & -1 & 1 & 0 & 2 & 1 & -1 \\
\hline a'c' $^{\prime}$ & -1 & 0 & 1 & 1 & 2 & 1 \\
\hline b'c' & 0 & -1 & 1 & -1 & 1 & 2 \\
\hline
\end{tabular}

As the problem addressed here is quite simple, it is possible to carry out the calculation of the $\mathbf{G f}$ matrix using the Chasles relation (13). For more complicated cases other techniques exist [20] to solve the Gf matrix constrained by complex geometrical or topological requirements.

\section{Vectorial association of initial and final configurations}

In order to respect the fixed point hypothesis for points $A, B$ and $C$ the Cholesky factorisation has been chosen. This criterion is only applicable to a metric tensor such as that defined in section 3.4. In order to obtain a metric tensor from the $\mathbf{G i}$ and $\mathbf{G f}$ Gram matrices, it is necessary to choose three independent vectors in the configuration to build an ortho-normal basis for the application of relation (8). As the Cholesky factorisation algorithm is recursive (see in [21]) the choice of the three vectors and their order is an important issue. In order to respect the zero expansion condition of the wall (see in section 4.1) the first two vectors are chosen as part of the wall and the third is just chosen to be independent from the two others. Finally the vectors $\mathbf{a b}$, ac and oa and a'b', a'c' and o'a' have been chosen respectively for initial and final configurations. The following metric tensors are then calculated. Mi and $\mathbf{M f}$ are associated respectively to the basis $\{\mathbf{a b}, \mathbf{a c}, \mathbf{o} \mathbf{a}\}$ and $\left\{\mathbf{a}^{\prime} \mathbf{b}\right.$ ' , a'c' , o'a'\} for the initial and final configurations. The $\mathbf{M i}$ and $\mathbf{M f}$ metric tensor are extracted from $\mathbf{G i}$ and $\mathbf{G}$ respectively.

These two metric tensors are then factorised with the Cholesky technique and the Mif metric tensor is calculated thanks to relation (10). Mif values are presented in table 6 below.

\begin{tabular}{|c|c|c|c|}
\hline & a'b' $^{\prime}$ & a'c' $^{\prime}$ & o'a' \\
\hline $\mathbf{a b}$ & 2 & 1 & -1 \\
\hline $\mathbf{a c}$ & 1 & 2 & -1 \\
\hline $\mathbf{o a}$ & -1 & -1 & 1.0024 \\
\hline
\end{tabular}

Table 6: Mif metric tensor

Again with the Chasles relation it is possible to deduce from Mif the global Gif matrix presented in table 7 below.

\begin{tabular}{|c|c|c|c|c|c|c|}
\hline & $\mathbf{o ' a}^{\prime}$ & $\mathbf{o ' b}^{\prime}$ & $\mathbf{o ' c}^{\prime}$ & $\mathbf{a}^{\prime} \mathbf{b}^{\prime}$ & $\mathbf{a}^{\prime} \mathbf{c}^{\prime}$ & $\mathbf{b}^{\prime} \mathbf{c}$ \\
\hline oa & 1,0024 & 0,0024 & 0,0024 & -1 & -1 & 0 \\
\hline ob & 0.0024 & 1.0024 & 0.0024 & 1 & 0 & -1 \\
\hline oc & 0.0024 & 0.0024 & 1.0024 & 0 & 1 & 1 \\
\hline ab & -1 & 1 & 0 & 2 & 1 & -1 \\
\hline ac & -1 & 0 & 1 & 1 & 2 & 1 \\
\hline bc & 0 & -1 & 1 & -1 & 1 & 2 \\
\hline
\end{tabular}

Table 7: Gif Gram matrix

As a first result we can notice that in Gif (table 7) the last three columns are exactly the same as in $\mathbf{G i}$ (table 3 ). This means that the position of points $A, B$ and $C$ has not changed between the initial and the final stages of the experiment.

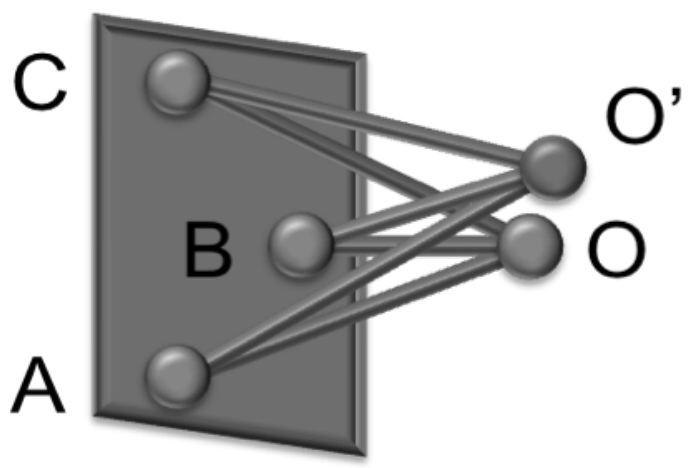

Figure 3: Initial and final configuration of the structure Finally the global matrix $\mathbf{G}$ representing the two configurations (figure 3 ) is obtained with the aggregation of $\mathbf{G i}, \mathbf{G f}$ and $\mathbf{G i f}$ described in figure 4 below.

$$
\mathbf{G}=\begin{array}{|c|c|}
\hline \mathbf{G i} & \text { Gif } \\
\hline \mathbf{G i f}^{\prime} & \mathbf{G f} \\
\hline
\end{array}
$$

Figure 4: global matrix $\mathbf{G}$ of the two configurations Geometrical requirement calculation:

In this application case, the geometrical requirement studied is the norm of the displacement vector for vertex $\mathrm{O}$. This displacement is defined by the vector oo'. Thanks to the Chasles relation the expression (15) is obtained.

$o o^{\prime}=0 a+a a^{\prime}+a^{\prime} o^{\prime}$

As explained in section Erreur! Source du renvoi introuvable. an affine association of the two configurations is required for the calculation of a vertex displacement. In this case it has been decided that the point $A$ and $A^{\prime}$ are coincident (others connection points such as the middle of a bar or the barycentre of $A, B, C$ could have been chosen). This means that the aa' vector is null. From there, the equation (15) becomes, after simplification, the relation (16).

oo' = oa - o'a' 
As $\left\|00^{\prime}\right\|=\sqrt{\left\langle 00^{\prime}, 00^{\prime}\right\rangle}$, the following paragraph will detail the calculation of $\left\langle\mathbf{0 0}^{\prime}, \mathbf{O o}^{\prime}\right\rangle$. With Chasles relation (16) we obtain the expression (17).

<0o',00'>=<0a,oa> + <o'a',o'a'> - 2 <0a, o'a'>

With the definition of $\beta 00^{\prime}$ 'such as in (18), relation (17) is rewritten as relation (19) using the principles set forth in relations (2) and (3).

$\beta_{00}^{\prime}=[1,0,0,0,0,0,-1,0,0,0,0,0]$

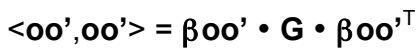

The coordinates of $\beta 0^{\prime}$ ' are directly deduced from relation (16), using the set of vectors Suif $=\left\{0 a, o b, o c, a b, a c, b c, o^{\prime} a^{\prime}, o^{\prime} b^{\prime}, o^{\prime} c^{\prime}, a^{\prime} \mathbf{b}^{\prime}, \mathbf{a}^{\prime} \mathbf{c}^{\prime}, \mathbf{b}^{\prime} \mathbf{c}^{\prime}\right\}$

defined accordingly with expression (5). One can notice that the $\beta$ oo' coordinates are exactly the coefficient of the

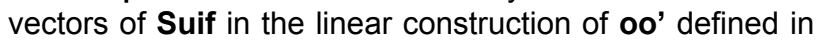
(16).

The numerical application of (19) gives the results expressed in equation (20).

$\left\|00^{\prime}\right\|=\sqrt{\left\langle 00^{\prime}, 00^{\prime}\right\rangle}=41 \mathrm{E}-4$.

In a manner similar to that of relation (18), the following vectors $(21,22,23)$ are defined for the calculation of the $<$ oa, 00'> < ob, 00'> and < oc, 00'> scalar products.

$\beta$ oa $=[1,0,0,0,0,0,0,0,0,0,0,0]$

$\beta$ ob $=[0,1,0,0,0,0,0,0,0,0,0,0]$

$\beta$ oc $=[0,0,1,0,0,0,0,0,0,0,0,0]$

For example the calculation of <0a, $\left.\mathbf{0 o}^{\prime}\right\rangle$ is given by relation (24) below.

$<0 a, 00^{\prime}>=\beta O a \cdot G \cdot \beta 00^{, \top}$

The numerical application of (24) gives the results expressed in (25) below.

$<$ oa, 0 ' $^{\prime}>=<$ ob,, o' $^{\prime}>=<$ oc, $00^{\prime}>=-2.4 \mathrm{E}-3$

The results presented in equations (20) and (25) prove that a coordinate free approach is applicable for the mapping of a geometrical requirement along the product life cycle.

\section{Verification using a Cartesian method}

As the application case is simple: only one vertex and three edges are subjected to some variations, the authors have applied a Cartesian method to solve problem and compare the result obtained with that of the previous section.

Let's suppose that the $O^{\prime}$ coordinates are $[x, y, z]$ in the initial global ortho-normal coordinate system $\{\mathbf{e i}, \mathbf{e j}, \mathbf{e k}\}$ of section 4.1. The coordinates of points $\{O, A, B, C\}$ are given in table 1. In order to determine the coordinates of $O^{\prime}$ after the thermal expansion, the following equations $(26,27$, 28) have to be solved:

\|o'a' $\|=\| \mathbf{o}^{\prime} \mathbf{a} \|=\sqrt{(1-x)^{2}+y^{2}+z^{2}}=1.0024$

$\left\|\mathbf{o}^{\prime} \mathbf{b}^{\prime}\right\|=\left\|\mathbf{o}^{\prime} \mathbf{b}\right\|=\sqrt{x^{2}+(1-y)^{2}+z^{2}}=1.0024$

$\left\|\mathbf{o}^{\prime} \mathbf{c}^{\prime}\right\|=\|\mathbf{o} \mathbf{c}\|=\sqrt{x^{2}+y^{2}+(1-z)^{2}}=1.0024$

This system gives the following solutions: $\{x, y, z\}=\{-2.394 \mathrm{E}-3, \quad-2.394 \mathrm{E}-3, \quad-2.394 \mathrm{E}-3\} \quad$ and $\{x, y, z\}=\{0.66906,0.66906,0.66906\}$. The second solution has to be excluded because it changes the topology of the structure: for this solution, the O' point has to pass through the wall.

This result has to be compared with that obtained in (25) because $\{\mathbf{o a}, \mathbf{o b} \mathbf{o c}\}=\{\mathbf{e i}, \mathbf{e j}, \mathbf{e k}\}$ (see table 3 ). One can see that the two methods give the same results.

\section{CONCLUSION AND PERSPECTIVES}

This paper has first presented some mathematical models and tools for a coordinate free approach to represent mechanisms. This model describes how to represent the mechanism in the proposed coordinate free model, how to calculate the value of a geometrical requirement at a given stage of the product life-cyle and finally how to link two geometrical configurations to map the evolution of a corresponding geometrical requirement. Later on an application of this coordinate free approach has been presented on a simple 3D example. The simplicity of the chosen case allowed the authors to address it from the beginning to the end using simple formulations and calculations. It has also allowed a comparison and validation of the results with a Cartesian approach.

Globally, this paper has shown that a generic coordinate free approach is applicable for the analysis of a geometrical requirement evolution along the product life cycle.

As the approach is generic, authors propose to investigate an application of this method on more complicated cases such as mobile mechanisms and hyperstatic mechanisms or structures. It is also envisioned to use more efficient existing solvers to obtain the global matrix $\mathbf{G}$, which would then allow the specification of requirements based on multiple configurations.

\section{REFERENCES}

[1] SAMPER, S., and Giordano, M., 1998, "Taking into Account Elastic Displacements in 3D Tolerancing Models and Application", Journal of Materials Processing Technology, 78(1-3) pp. 156-162.

[2] SAMPER, S., and Giordano, M., 2003, "Simultaneous Analysis Method for Tolerancing Flexible Mechanisms", Geometric Product Specification and Verification: Integration of Functionality, pp. 127-134.

[3] CID, G., Thiebaut, F., Bourdet, P., 2007, "Geometrical Study of Assembly Behaviour, Taking into Accounts Rigid Components Deviations, Actual Geometric Variations and Deformations", Models for Computer Aided Tolerancing in Design and Manufacturing, pp. 301-310.

[4] CID, G., Thiebaut, F., and Bourdet, P., 2004, "Taking the deformation into account for components' tolerancing", 5th International Conference on Integrated Design and Manufacturing in Mechanical Engineering (IDMME 2004), LURPA, ENS de Cachan, Cachan, France; Univ. of Bath, Bath, UK, pp. 40.

[5] PIERRE, L., Teissandier, D., and Nadeau, J.P., 2008, "Tolerancing analysis taking into account thermomechanical strains", Proceedings of IDMME2008, Beijing, October 8-10, China.

[6] PIERRE L., Teissandier, D., and Nadeau J.P., 2009, "Integration of multiple physical behaviours into a geometric tolerancing approach", 11th CIRP International Seminar on Computer-Aided Tolerancing, Annecy. 
[7] DESROCHERS, A., 1991, "Modèle conceptuel du dimensionnement et du tolérancement des mécanismes. Représentation dans les systèmes CFAO.", Ecole Centrale Paris, PhD. thesis.

[8] DESROCHERS, A., 2003, "A CAD/CAM Representation Model Applied to Tolerance Transfer Methods", Journal of Mechanical Design, Transactions of the ASME, 125(1) pp. 14-22.

[9] CLEMENT, A., Valade, C., and Riviere, A., 1997, "The TTRSs: 13 oriented constraints for dimensioning, tolerancing and inspection", Proceedings of Euroconference: Advanced Mathematical Tools in Metrology III, World Scientific, Berlin, Germany, pp. 24-42.

[10] SERRÉ, P., 2000, "Cohérence de la spécification d'un objet de l'espace euclidien à n dimensions", Ecole Centrale Paris, PhD. thesis.

[11] http://en.wikipedia.org/wiki/Gramian_matrix

[12] ZINA, S., Lombard, M., Lussent, L., 2006, "Generic modeling and configuration management in product lifecycle management", IMACS Multiconference on "Computational Engineering in Systems Applications", CESA, October 04,2006 - October 06, Inst. of Elec. and Elec. Eng. Computer Society, Beijing, China, pp. 1252-1258.

[13] EYNARD, B., Gallet, T., Nowak, P., 2004, "UML Based Specifications of PDM Product Structure and Workflow", Computers in Industry, 55(3) pp. 301.

[14] MANDIL, G., Desrochers, A., and Rivière, A., 2009, "Computational Methodology for the Prediction of Functional Requirement Variations Across the Product Life-Cycle", 11th CIRP International Seminar on Computer-Aided Tolerancing, Annecy, http://hal.archives-ouvertes.fr/hal-00381625/fr/.

[15] MANDIL, G., Desrochers, A., and Rivière, A., 2009, "Framework for the Monitoring of Functional Requirements Along the Product Life Cycle", Proceedings of the 6th Conference of Conception \& Production Intégrées, http://hal.archives-ouvertes.fr/hal-00426416/fr/.

[16] ANSELMETTI, B., 2005, "Génération automatique de la cotation fonctionnelle par la méthode CLIC".

[17] ANSELMETTI, B., 2006, "Generation of Functional Tolerancing Based on Positioning Features", Computer-Aided Design, 38(8) pp. 902-919.

[18] GHIE, W., 2004, " Modèle unifié Jacobien-Torseur pour le tolérancement assisté par ordinateur ", Université de Sherbrooke, PhD. Thesis.

[19] GHIE, W., Desrochers, A., and Laperrière, L., 2007, "Assessment of design parameter space using tolerance analysis", 10th International Seminar On Computer Aided Tolerancing, CIRP.

[20] MOINET, M., 2009, "Descriptions non cartésiennes et résolution de problèmes géométriques sous contraintes", Ecole Centrale Paris, PhD. thesis.

[21] http://fr.wikipedia.org/wiki/Factorisation_de_Cholesky 